\title{
ДИНАМИКА КЛИНИКО-ЛАБОРАТОРНЫХ ПОКАЗАТЕЛЕЙ У ДЕТЕЙ ПРИ ПРОВЕДЕНИИ ВНУТРИВЕННОЙ АНЕСТЕЗИИ В СТОМАТОЛОГИИ
}

Использование общей анестезии в амбулаторной анестезиологии (стоматологии) связано прежде всего с проблемой дентофобии. Среди всех возрастных групп пациентов наибольшую сложность представляет детский контингент. Дентофобия перед стоматологическим лечением считается одной из самых больших проблем как для стоматолога, так и для пациентов, особенно детей. Более того, страх лечения зубов по-прежнему считается основным препятствием для проведения полноценного стоматологического лечения [1]. Эта проблема сделала почти невозможным лечение детей от 1 года до 4 лет без использования общей анестезии или седации. Несмотря на все факторы, прием детей в клиниках для лечения зубов с использованием общей анестезии увеличивается с каждым годом [2] Использование седативных препаратов может быть полезным для лечения дентофобии у некоторых пациентов [3]. Поэтому важно обеспечить, чтобы индукция, течение анестезии и пробуждение были безопасны и комфортны. Индукция анестезии затрагивает не только детей, но также может вызывать стрессы у родителей. Следовательно, родители играют важную роль в поддержке своих детей во время стоматологического лечения детей. [4]

\section{ЦЕЛ Ь РАБОТ Ы}

Выявление особенностей клиниколабораторных данных и маркеров адекватности проводимой внутривенной анестезии пропофолом у детей.

\section{МАТЕРИАЛ И МЕТОДЫ}

Исследование проводилось в детской стоматологической клинике «Дентал Гуру» г. Донецка.

В период с ноября 2018 года по май 2020 было набрано 220 детей, в возрасте от 1,5 до 10 лет, проходящих комплексное лечение зубов под общей анестезией. По возрасту, от 1,5 до 3 лет - 92
(42\%) пациента, от 4 до 6 лет - 86 (39,3\%) пациентов, от 7 до 10 лет - 41 (18,7\%) пациент. Одному ребенку было отказано в лечении под общей анестезией пропофолом в связи с синдромом Вилсона. Вследствие ОРЗ пять пациентов были перенесены на более поздний срок лечения. Критерии исключения включали любые острые инфекционные заболевания (включая ОР3), недавнюю вакцинацию (не менее недели), хронические заболевания верхних и нижних дыхательных путей в стадии обострения, аллергию на препараты, применяемые для общей анестезии. Всего в исследование было включено 219 детей.

В обязательном порядке всем пациентам проводились обследования:

1. Общий анализ крови.

2. Общий анализ мочи.

3. Биохимический анализ крови.

4. ЭКГ.

5. Заключение педиатра.

Предоперационное отсутствие приема пищи у детей сводилось к 6-часовому периоду голодания, прозрачные жидкости (вода, чай, фруктовый сок) - за 2 часа до анестезии. У родителей или опекунов всех участников исследования было получено письменное информированное согласие на проведение лечения под общей анестезией.

Премедикация сводилась к принципу «минимум уколов». Если предоставляется возможность провести пероральную премедикацию, то она более предпочтительна, чем внутримышечная. Несмотря на то, что атропин в данный момент выведен из списка «стандартной» премедикации, в стоматологии имеются некоторые особенности: инструментальное раздражение рефлексогенных зон, необходимость избегать выраженной саливации и возможной бра-

(c) А.Н. Колесников, А.А. Алексеенко, 2020

(c) Университетская Клиника, 2020 


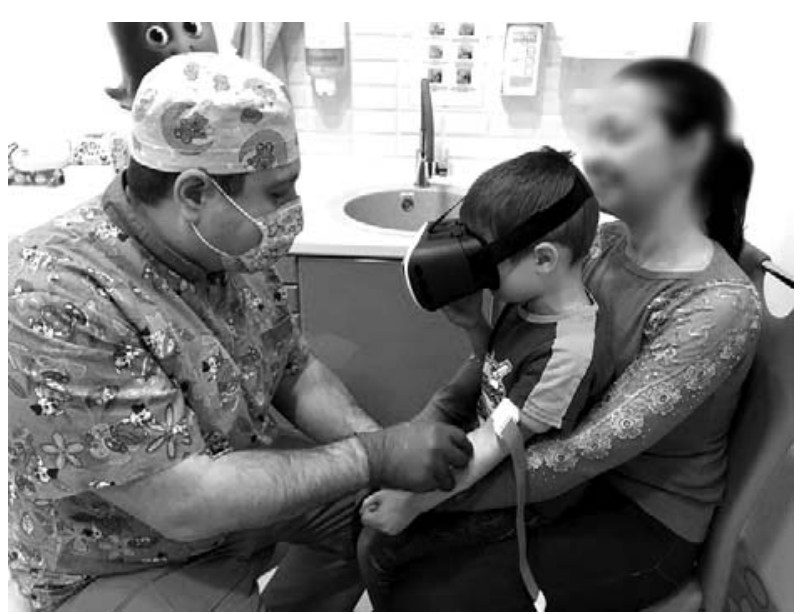

Рис. 1. Катетеризация периферической вены мальчику 5 лет, в то время как ребенок смотрит мультфильм в очках виртуальной реальности.

дикардии. В премедикацию назначалось внутривенное введение атропина и дексаметазона (табл. 1.)

При оценке статистических данных в различных группах по возрасту выявлено, что, как правило, атропин $0,1 \%$ вводился у детей $1-3$ лет в дозировке $0,17 \pm 0,03$ мл, у детей 4-6 лет - 0,21 $\pm 0,08$ мл, у детей 7-10 лет - 0,31 $\pm 0,04$ мл; дексаметазон у детей 1-3 лет - $1,86 \pm 0,23$ мг, у детей 4-6 лет

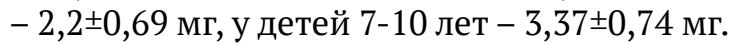

При невозможности наладить контакт с «трудным» в психосоматическом плане ребенком перед анестезией можно назначить перорально сироп мидазолама в дозе 0,5 мг/кг или мидазолам внутримышечно в дозе 0,05-0,15 мг/ кг. Также довольно эффективно использовался способ немедикаментозного отвлечения внимания ребенка от катетеризации периферической вены. После орошения места пункции 10-процентным раствором лидокаина ребенку предлагалось посмотреть мультфильм в очках виртуальной реальности, в то время как произ-

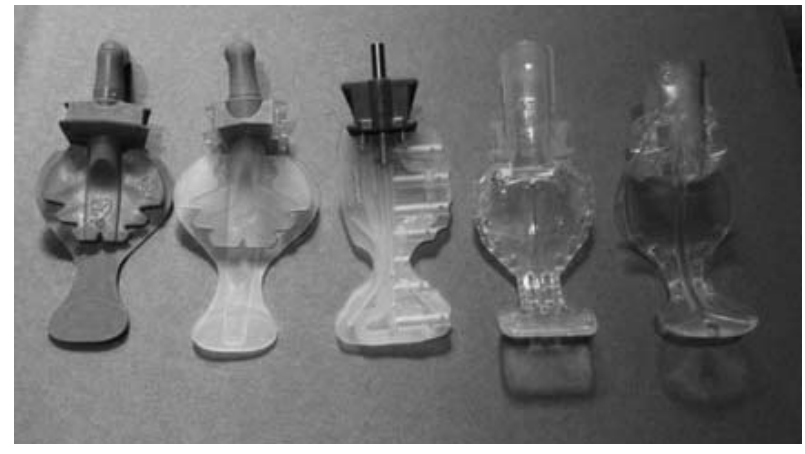

Рис. 2. Различные эвакуационные системы.

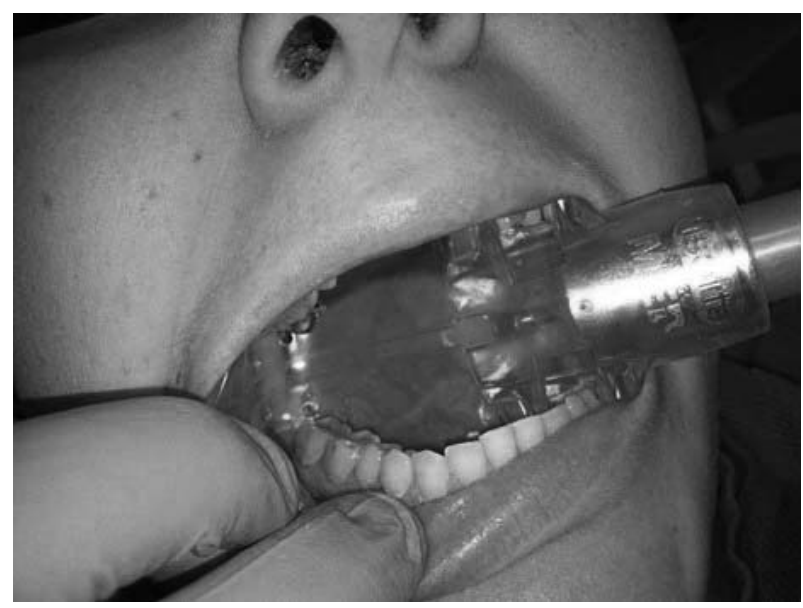

Рис. 3. Эвакуационная система Isolite при лечении зубов у девочки 14 лет.

водилась внутривенная катетеризация и индукция пропофолом (рис. 1.).

Объем интраоперационного мониторинга включал [6]:

- электрокардиографию с подсчетом ЧСС;

- пульсовую оксиметрию;

- измерение АД неинвазивным методом;

- термометрию;

Дозировка препаратов для премедикации

Таблица 1.

\begin{tabular}{|c|c|c|c|}
\hline $\begin{array}{l}\text { Возраст } \\
\text { детей }\end{array}$ & Препарат & $\begin{array}{c}\text { Число } \\
\text { наблюдений } \\
\end{array}$ & $\begin{array}{c}\mathrm{M} \pm \mathrm{sd} \\
(\min -\max )\end{array}$ \\
\hline \multirow{2}{*}{ 1-3 года } & Премедикация атропин 0,1\% (мл) & 92 & $\begin{array}{l}0,17 \pm 0,03 \\
(0,1-0,2)\end{array}$ \\
\hline & Премедикация дексаметазон (мг) & 92 & $\begin{array}{l}1,86 \pm 0,23 \\
(1,5-2,0)\end{array}$ \\
\hline \multirow{2}{*}{ 4-6 лет } & Премедикация атропин 0,1\% (мл) & 86 & $\begin{array}{l}0,21 \pm 0,08 \\
(0,15-0,4)\end{array}$ \\
\hline & Премедикация дексаметазон (мг) & 86 & $\begin{array}{c}2,2 \pm 0,69 \\
(1,5-4,0)\end{array}$ \\
\hline \multirow{2}{*}{ 7-10 лет } & Премедикация атропин 0,1\% (мл) & 41 & $\begin{array}{l}0,31 \pm 0,04 \\
(0,3-0,4)\end{array}$ \\
\hline & Премедикация дексаметазон (мг) & 41 & $\begin{array}{l}3,37 \pm 0,74 \\
(2,0-4,0)\end{array}$ \\
\hline
\end{tabular}


Средние дозы пропофола и объем инфузии

Таблица 2.

\begin{tabular}{lcc}
\hline \multicolumn{1}{c}{ Препараты } & Число наблюдений & $\begin{array}{c}\text { M } \pm \text { sd } \\
(\min -\max )\end{array}$ \\
\hline \hline Инфузия кристаллоидов (мл) & 219 & $\begin{array}{c}227,90 \pm 62,96 \\
(200,0-400,0)\end{array}$ \\
\hline Индукция пропофол 1\% (мл) & 219 & $\begin{array}{c}3,81 \pm 1,13 \\
(2,0-7,0)\end{array}$ \\
\hline Поддержание анестезии пропофол 1\% (мл) & 219 & $\begin{array}{c}33,51 \pm 11,71 \\
(12,0-60,0)\end{array}$ \\
\hline
\end{tabular}

Описательная статистика лабораторных данных у детей

Таблица 3.

\begin{tabular}{lcc}
\hline \multicolumn{1}{c}{ Лабораторные данные } & Число наблюдений & $\begin{array}{c}\mathrm{M} \pm \mathrm{Sd} \\
(\min -\max )\end{array}$ \\
\hline \hline эритроциты (Т/л) & 219 & $\begin{array}{c}4,55 \pm 0,48 \\
(3,6-5,6)\end{array}$ \\
\hline гемоглобин (Г/л) & 219 & $\begin{array}{c}131,21 \pm 11,46 \\
(104,0-162,0)\end{array}$ \\
\hline тромбоциты (Г/л) & 219 & $\begin{array}{c}289,63 \pm 61,56 \\
(195,0-542,0)\end{array}$ \\
\hline лейкоциты (Г/л) & 219 & $\begin{array}{c}7,0 \pm 1,86 \\
(4,0-13,0)\end{array}$ \\
\hline СОЭ (мм/ч) & 219 & $\begin{array}{c}4,74 \pm 2,42 \\
(2,0-15,0)\end{array}$ \\
\hline палочкоядерные нейтрофилы (\%) & 219 & $\begin{array}{c}1,76 \pm 1,16 \\
(1,0-7,0)\end{array}$ \\
\hline сегментоядерные нейтрофилы (\%) & 219 & $\begin{array}{c}36,79 \pm 9,36 \\
(24,0-58,0)\end{array}$ \\
\hline эозинофилы (\%) & 219 & $\begin{array}{c}2,84 \pm 1,92 \\
(0,0-10,0)\end{array}$ \\
\hline лимфоциты (\%) & 219 & $\begin{array}{c}52,11 \pm 8,82 \\
(31,5-65,0)\end{array}$ \\
\hline моноциты (\%) & 219 & $\begin{array}{c}6,80 \pm 2,33 \\
(1,0-11,0)\end{array}$ \\
\hline
\end{tabular}

- капнографию с определением содержания $\mathrm{CO}_{2}$ в конце выдоха $\left(\mathrm{EtCO}_{2}\right)$;

- контроль ЧД.

Особенности проведения анестезии у стоматологических больных обусловлены в первую очередь локализацией операционного поля в непосредственной близости от верхних дыхательных путей. В практике лечения зубов, особенно если выключение сознания ребенка обязательно, вопрос о выборе средств поддержания проходимости дыхательных путей остается очень важным. Также необходимо заранее прогнозировать и быть готовым к ряду осложнений как во время манипуляции, так и после нее. Нами было использована эвакуационная системa Isolite (рис. 2. и рис. 3.), которая обладает рядом преимуществ: эвакуация жидкости и пыли производится постоянно и равномерно со всей полости рта без участия ассистента хирурга, что позволяет ассистенту уделить больше внимания проведению самого лечения; наличие накусочного блока, который позволяет зафиксировать широту открытия рта на длительное время, несмотря на уровень сознания пациента; просто- та использования и освещение рабочей зоны. Основным недостатком данной системы является отсутствие герметичности дыхательных путей [5].

После установки внутривенного катетера препаратом для проведения внутривенной индукции являлся пропофол. Индукционная доза составляла $38,1 \pm 11,3$ мг. Анестезия поддержива-

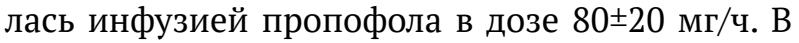
таблице 2 представлены данные по дозировкам пропофола и объему инфузионной терапии.

Обезболивание во время лечения проводилось с помощью местной инфильтрационной и проводниковой анестезии в полном объеме. Использовался артикаин с содержанием адреналина $1: 200000$.

Инфузия кристаллоидов обеспечивала физиологические потребности в жидкости и восполнения ее дефицита, образовавшегося в результате предоперационного голодания.

Ребенок оставался в клинике под наблюдением анестезиолога в течение необходимого для полного пробуждения времени (чаще всего - до 1 часа). Послеоперационный контроль включал 
Динамика изменений показателей на этапах лечения

Таблица 4.

\begin{tabular}{lcccc}
\hline \multicolumn{1}{c}{ Показатели } & $\begin{array}{c}\text { За } 10 \text { мин до } \\
\text { лечения }\end{array}$ & Начало лечения & $\begin{array}{c}\text { Базисная ане- } \\
\text { стезия }\end{array}$ & Пробуждение \\
\hline \hline $\mathrm{SpO}_{2}$ (\%) & $98,58 \pm 0,63$ & $97,51 \pm 0,59$ & $97,69 \pm 1,4$ & $98,5 \pm 0,7$ \\
\hline РеtCO (мм рт. ст.) & $38,3 \pm 0,8$ & $38,1 \pm 0,88$ & $38,3 \pm 0,84$ & $37,5 \pm 0,7$ \\
\hline среднее АД (мм рт. ст.) & $68,9 \pm 4,6$ & $70,1 \pm 4$ & $69,1 \pm 3,8$ & $70 \pm 2,8$ \\
\hline ЧСС (в мин) & $102,4 \pm 7,8$ & $107 \pm 7,9$ & $106,7 \pm 7,7$ & $99,5 \pm 0,7$ \\
\hline ЧДД (в мин) & $22,5 \pm 2$ & $22,5 \pm 1,9$ & $21,7 \pm 1,6$ & $22,5 \pm 0,7$ \\
\hline
\end{tabular}

Ранговые корреляции Спирмена. (корреляции на уровне $\mathrm{p}<0,05$ )

Таблица 5.

\begin{tabular}{|c|c|c|c|}
\hline Пара переменных & Число набл. & Спирмена R & $\mathrm{p}$ \\
\hline эритроциты \& ЧСС начало лечения & 219 & 0,33 & 0,03 \\
\hline эритроциты \& ЧСС базисная анестезия & 219 & 0,30 & 0,05 \\
\hline гемоглобин \& премедикация атропина & 219 & 0,32 & 0,03 \\
\hline гемоглобин \& поддержка анестезии пропофолом & 219 & 0,34 & 0,03 \\
\hline тромбоциты \& длительность кровотечения & 219 & 0,32 & 0,04 \\
\hline тромбоциты \& SpO2 базисная анестезия & 219 & $-0,33$ & 0,03 \\
\hline тромбоциты \& ЧСС начало лечения & 219 & 0,30 & 0,05 \\
\hline тромбоциты \& PetCO2 начало лечения & 219 & $-0,49$ & 0,001 \\
\hline лейкоциты \& длительность кровотечения & 219 & 0,32 & 0,04 \\
\hline палочкоядерные нейтрофилы \& индукция пропофола & 219 & $-0,34$ & 0,03 \\
\hline палочкоядерные нейтрофилы \& SpO2 базисная анестезия & 219 & 0,38 & 0,01 \\
\hline палочкоядерные нейтрофилы \& ЧСС базисная анестезия & 219 & 0,47 & 0,01 \\
\hline сегментоядерные нейтрофилы \& ср. АД базисная анестезия & 219 & 0,35 & 0,03 \\
\hline сегментоядерные нейтрофилы \& ЧДД базисная анестезия & 219 & $-0,38$ & 0,02 \\
\hline сегментоядерные нейтрофилы \& PetCO2 до лечения & 219 & $-0,31$ & 0,04 \\
\hline лимфоциты \& ср. АД базисная анестезия & 219 & $-0,33$ & 0,03 \\
\hline лимфоциты \& ЧДД базисная анестезия & 219 & 0,32 & 0,05 \\
\hline лимфоциты \& PetCO2 до лечения & 219 & 0,33 & 0,03 \\
\hline
\end{tabular}

в себя оценку общего состояния ребенка, возможности установления вербального контакта, адекватности самостоятельного дыхания, стабильности гемодинамики, степени выраженности болевого синдрома, контроль кровотечения из лунок удаленных зубов, наличия психомоторного возбуждения, тошноты и рвоты.

После общения с родителями и разъяснения их поведения в послеоперационном периоде ребенка отпускали домой. По договоренности через 1-2 ч и 24 ч после ухода ребенка из клиники осуществлялся контрольный телефонный звонок родителям с целью уточнения его состояния.

\section{РЕЗУЛЬТАТЫ И ОБСУЖДЕНИЕ}

В результате лабораторного обследования пациентов были получены данные, представленные в таблице 3.

Отклонения лабораторных данных от нормы незначительное. У 48 детей отмечалось увеличение количества эозинофилов в связи с различными аллергическими проявлениями.

Мониторинг внутривенной анестезии пропофолом проводился постоянно по Гарвардско- 
му стандарту мониторинга для общей анестезии (табл. 4.).

Проведение внутривенной анестезии пропофолом у детей с обеспечением проходимости дыхательных путей эвакуационной системой «Isolite» на всех этапах лечения отличается стабильными показателями гемодинамики, PetCO2, SpO2 - в пределах физиологической и возрастной нормы.

В ходе данного исследования впервые были оценены корреляционные зависимости предоперационных лабораторных данных и предоперационные, интраоперационные и послеоперационные показатели мониторинга (табл. 5.).

При оценке ранговых корреляций Спирмена обращает на себя внимание прямо пропорциональная зависимость количества гемоглобина и количества введенного для поддержания анестезии пропофола (p=0,026); увеличение количества палочкоядерных нейтрофилов приводит к уменьшению дозы пропофола на индукцию $(\mathrm{p}=0,025)$ и увеличению $\mathrm{SpO} 2$ во время лечения $(\mathrm{p}=0,013) ;$ увеличение сегментоядерных нейтрофилов приводит к увеличению ЧДД во время анестезии $(\mathrm{p}=0,016)$ и увеличению PetCO2 до лечения $(\mathrm{p}=0,044)$; увеличение числа лимфоцитов приводит к увеличению PetCO2 до лечения $(\mathrm{p}=0,029)$. Так же и обратное изменение лабораторных показателей приводит к обратным изменениям показателей на этапах лечения.

\section{З А К ЛЮ Ч Н ИЕ}

Оценка общего клинического анализа крови у детей является не только рутинной, закрепленной протоколами процедурой, но может помочь в трактовке клинических данных (и их изменений), таких как SpO2, PetCO2, ЧДД и гемодинамика, а также прогнозировать течение внутривенной анестезии пропофолом у детей.

По данным проведенного исследования можно сказать, что комбинированная анестезия у детей (инфильтрационное обезболивание артикаином и седация пропофолом) отличается стабильностью клинических показателей на всех этапах стоматологического лечения и отсутствием осложнений при условии применения эвакуационной системы Isolite.

\section{А.Н. Колесников, А.А. Алексеенко}

ГОО ВПО «Донецкий национальный медицинский университет имени М. Горького», Донецк

\section{ДИНАМИКА КЛИНИКО-ЛАБОРАТОРНЫХ ПОКАЗАТЕЛЕЙ У ДЕТЕЙ ПРИ ПРОВЕДЕНИИ ВНУТРИВЕННОЙ АНЕСТЕЗИИ В СТОМАТОЛОГИИ}

Использование общей анестезии в амбулаторной анестезиологии (стоматологии) связано прежде всего с проблемой дентофобии. Среди всех возрастных групп пациентов наибольшую сложность представляет детский контингент. Целью данного исследования было выявить особенности клинико-лабораторных данных и маркеров адекватности проводимой внутривенной анестезии пропофолом у детей. Исследование проводилось в детской стоматологической клинике «Дентал Гуру» г. Донецка. В период с ноября 2018 года по май 2020 было включено 219 детей от 1,5 до 10 лет. При оценке статистических данных в различных группах по возрасту выявлено, что, как правило, атропин 0,1\% вводился у детей 1-3 лет в дозировке $0,17 \pm 0,03$ мл, у детей 4-6 лет $-0,21 \pm 0,08$ мл, у детей 7-10 лет - 0,31 $\pm 0,04$ мл; дексаметазон у детей 1-3

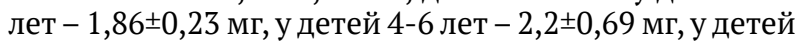

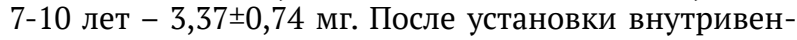
ного катетера препаратом для проведения внутривенной индукции являлся пропофол. Индукционная доза составляла $38,1 \pm 11,3$ мг. Анестезия поддерживалась инфузией пропофола в дозе $80 \pm 20$ мг/ч. В ходе данного исследования впервые были оценены корреляционные зависимости предоперационных лабораторных данных и предоперационные, интраоперационные и послеоперационные показатели мониторинга. При оценке ранговых корреляций Спирмена обращают на себя внимание некоторые корреляционные зависимости. Оценка общего клинического анализа крови у детей является не только рутинной, закрепленной протоколами процедурой, а но может помочь в трактовке клинических данных (и их изменений), таких как SpO2, PetCO2, ЧДД и гемодинамика, а также прогнозировать течение внутривенной анестезии пропофолом у детей.

По данным проведенного исследования можно сказать, что комбинированная анестезия у детей (инфильтрационное обезболивание артикаином и седация пропофолом) отличается стабильностью клинических показателей на всех этапах стоматологического лечения и отсутствием осложнений при условии применения эвакуационной системы Isolite.

Ключевые слова: анестезиология, клиниколабораторные данные, седация, дентофобия. 


\section{A.N. Kolesnikov, A.A. Alexeenko}

\section{SEI HPE «M. Gorky Donetsk National Medical University», Donetsk}

\section{DYNAMICS OF CLINICAL LABORATORY INDICATORS IN CHILDREN WITH THE CONDUCT OF INTRAVENOUS ANESTHESIA IN DENTISTRY}

The use of general anesthesia in outpatient anesthesiology (dentistry) is primarily associated with the problem of dentophobia. Among all age groups of patients, the greatest difficulty is the children's contingent. The purpose of this study was to identify the features of clinical and laboratory data and markers of the adequacy of intravenous anesthesia with propofol in children. The study was carried out in the children's dental clinic "Dental Guru" in Donetsk. In the period from November 2018 to May 2020, 219 children from 1.5 to 10 years old were included. When evaluating the statistical data in different age groups, it was found that, as a rule, atropine $0.1 \%$ was administered in a dosage of $0.17 \pm 0.03 \mathrm{ml}$ in children $1-3$ years old, $0.21 \pm 0,08 \mathrm{ml}$ in children $4-6$ years old, in children $7-10$ years old $-0.31 \pm 0.04 \mathrm{ml}$; dexamethasone in children $1-3$ years old $1.86 \pm 0.23 \mathrm{mg}$, in children $4-6$ years old $-2.2 \pm 0.69 \mathrm{mg}$, in children $7-10$ years old $-3.37 \pm 0.74$ $\mathrm{mg}$. After the placement of an intravenous catheter, the drug for intravenous induction was propofol. The induction dose was $38.1 \pm 11.3 \mathrm{mg}$. Anesthesia was maintained with propofol infusion at a dose of $80 \pm 20 \mathrm{mg} / \mathrm{h}$. In the course of this study, the correlation dependences of preoperative laboratory data and preoperative, intraoperative and postoperative monitoring indicators were evaluated for the first time. When assessing Spearman's rank correlations, some correlations are noteworthy. Evaluation of a CBC in children is not only a routine protocolbased procedure, but can help in the interpretation of clinical data (and their changes), such as SpO2, PetCO2, NPV and hemodynamics. Also predict the course of intravenous anesthesia with propofol in children. According to the study, it can be said that combined anesthesia in children (infiltration anesthesia with articaine and sedation with propofol) is characterized by the stability of clinical parameters at all stages of dental treatment and the absence of complications, provided that the Isolite evacuation system is used.

Key words: anesthesiology, clinical laboratory data, sedation, a crippling dental phobia.

\section{ЛИТЕРАТУРА}

1. Newton JT. Interpreting pain as 'catastrophic' makes it worse: The neurological basis. J Dent Res. 2013;92:107108.

2. Welbury R. Summary of: Why are children still having preventable extractions under general anaesthetic? A service evaluation of the views of parents of a high caries risk group of children. Br Dent J. 2011;210:360-361.

3. Gazal G, Fareed WM, Zafar MS, Al-Samadani KH. Pain and anxiety management for pediatric dental procedures using various combinations of sedative drugs. Saudi Pharm J. 2016; 24 (4): 379-385. doi: 10.1016/j.jsps.2014.04.004.

4. Peterson L, Shigetomi C. The use of coping techniques to minimize anxiety in hospitalized children. Behav Ther. $1981 ; 12: 1-14$

5. Dahlke WO1, Cottam MR, Herring MC, Leavitt JM, Ditmyer MM, Walker RS. Evaluation of the spatter-reduction effectiveness of two dry-field isolation techniques. J Am Dent Assoc. 2012; 143 (11): 1199-1204.

6. Association of Anaesthetists of Great Britain and Ireland. Recommendations for standards of monitoring during anaesthesia and recovery 2015. Anaesthesia. 2016; 71: 8593.

\section{REFERENCES}

1. Newton JT. Interpreting pain as 'catastrophic' makes it worse: The neurological basis. J Dent Res. 2013;92:107108.

2. Welbury R. Summary of: Why are children still having preventable extractions under general anaesthetic? A service evaluation of the views of parents of a high caries risk group of children. Br Dent J. 2011;210:360-361.

3. Gazal G, Fareed WM, Zafar MS, Al-Samadani KH. Pain and anxiety management for pediatric dental procedures using various combinations of sedative drugs. Saudi Pharm J. 2016; 24 (4): 379-385. doi: 10.1016/j.jsps.2014.04.004.

4. Peterson L, Shigetomi C. The use of coping techniques to minimize anxiety in hospitalized children. Behav Ther. 1981;12:1-14.

5. Dahlke WO1, Cottam MR, Herring MC, Leavitt JM, Ditmyer MM, Walker RS. Evaluation of the spatter-reduction effectiveness of two dry-field isolation techniques. J Am Dent Assoc. 2012; 143 (11): 1199-1204.

6. Association of Anaesthetists of Great Britain and Ireland. Recommendations for standards of monitoring during anaesthesia and recovery 2015. Anaesthesia. 2016; 71: 8593. 